\title{
Une nouvelle menace pour le Saumon sur le Bassin de la Loire : la chenalisation de l'estuaire
}

\author{
Robin Cuinat \\ Délégué Régional du Conseil Supérieur de la Pêche à Clermont-Ferrand \\ Région Auvergne-Limousin \\ Marmilhat, 63370 Lempdes
}

Parmi les cinq espèces de poissons migrateurs du Bassin de la Loire - Anguille, Lamproie, Alose, Truite de mer et Saumon atlantique - c'est le Saumon qui a fait l'objet, dans les vingt dernières années, des plus grands efforts de réhabilitation, sur l'Allier et certains affluents (Vienne-Creuse - Gartempe, Sioule, Dore, Allagnon). De nombreuses passes migratoires ont été réalisées, les repeuplements ont été intensifiés, et les règlements de pêche ont été de plus en plus restrictifs.

Malgré cela, les contingents annuels de saumons sont restés faibles. A cela, deux causes principales:

- la poursuite des pêches sur la zone de grossissement au large du Groënland;

- et, plus récemment, la transformation de l'estuaire, du fait des dragages opérés pour accueillir les gros navires très en amont de St-Nazaire. L'étendue du bouchon vaseux, dans lequel la teneur en oxygène peut tomber à zéro, est maintenant passée d'une dizaine à une trentaine de kilomètres, constituant un obstacle biologique total en dehors des périodes de crue.

La souche de Saumon Loire-Allier présente des caractéristiques uniques en Europe: longue période de croissance en mer (et grande taille au retour), et long séjour des adultes en eau douce (6 à 14 mois), adapté à la grande distance $(\approx 900 \mathrm{~km}$ ) entre l'estuaire et les frayères. Elle est sérieusement menacée de disparition si l'on ne remédie pas à la dégradation de l'estuaire.

Les autres espèces migratrices sont également affectées par cette dégradation, ainsi que divers poissons marins pour lesquels l'estuaire constituait une zone privilégiée de vie juvénile.

La Loire et une partie de ses affluents héberge cinq espèces de poissons grands migrateurs :

- l'une se reproduit en mer et effectue le plus gros de sa croissance en eau douce : l'Anguille ;

- les quatre autres font l'inverse : la Lamproie, l'Alose, la Truite de mer et le Saumon. C'est de ce dernier, à la fois le plus prestigieux et le plus menacé, que nous traiterons dans cet article, ce qui ne signifie pas que les autres migrateurs soient sans intérêt écologique ni économique*.

\section{Le cycle du saumon: un grand périple}

C'est dans la partie supérieure de cette rivière, où les eaux sont claires et rapides, que naissent les saumons. Au stade juvénile, ces «tacons " ressemblent à première vue à des truites. On les distingue à leur mâchoire plus petite, à leurs grandes pectorales, à leur queue plus fourchue et à leurs grandes taches sombres sur les flancs.

A l'âge d'un an et demi (parfois deux et demi) mesurant 14 à $20 \mathrm{~cm}$, ils prennent une robe argentée et dévalent, en avril et mai, vers l'Océan. On les appelle «saumoneaux» (ou « smolts").

La plupart des saumons Loire-Allier vont se nourrir entre le Groënland et le Canada, où ils se nourrissent essentiellement de poissons, et acquièrent leur chair rose. Deux ou trois ans plus tard, pesant de trois à plus de dix kilos, ils reviennent près de nos côtes. Pour naviguer en mer, ils s'orientent d'après les astres et probablement aussi d'après le champ magnétique terrestre.

En rivière, ils recherchent les odeurs qu'ils avaient mémorisées lors de leur dévalaison. Pendans leur remontée de la Loire, puis de l'Allier, qui nécessite de six à quatorze mois, ils ne prennent aucune nourriture. En été, la migration est ralentie, et même complètement suspendue sur le haut-Allier.

La reproduction a lieu en novembre-décembre: la femelle prépare un grand nid dans les galets, puis y dépose ses œufs. L'éclosion a lieu en fin d'hiver. Un à deux pour cent seulement des géniteurs survivront et reviendront frayer une deuxième fois.

Le cycle vital du Saumon Loire-Allier est de quatre à sept ans selon les individus; il est donc long et difficile d'évaluer les effets des mesures règlementaires sur des interventions sur le milieu ; ceci d'autant plus que les facteurs hydrologiques, très variables selon les années, jouent un rôle important sur la survie et les migrations.

* Un article traitant de l'ensemble des migrateurs du Bassin de la Loire a été publié fin 1991 dans la "Revue d'Auvergne". 


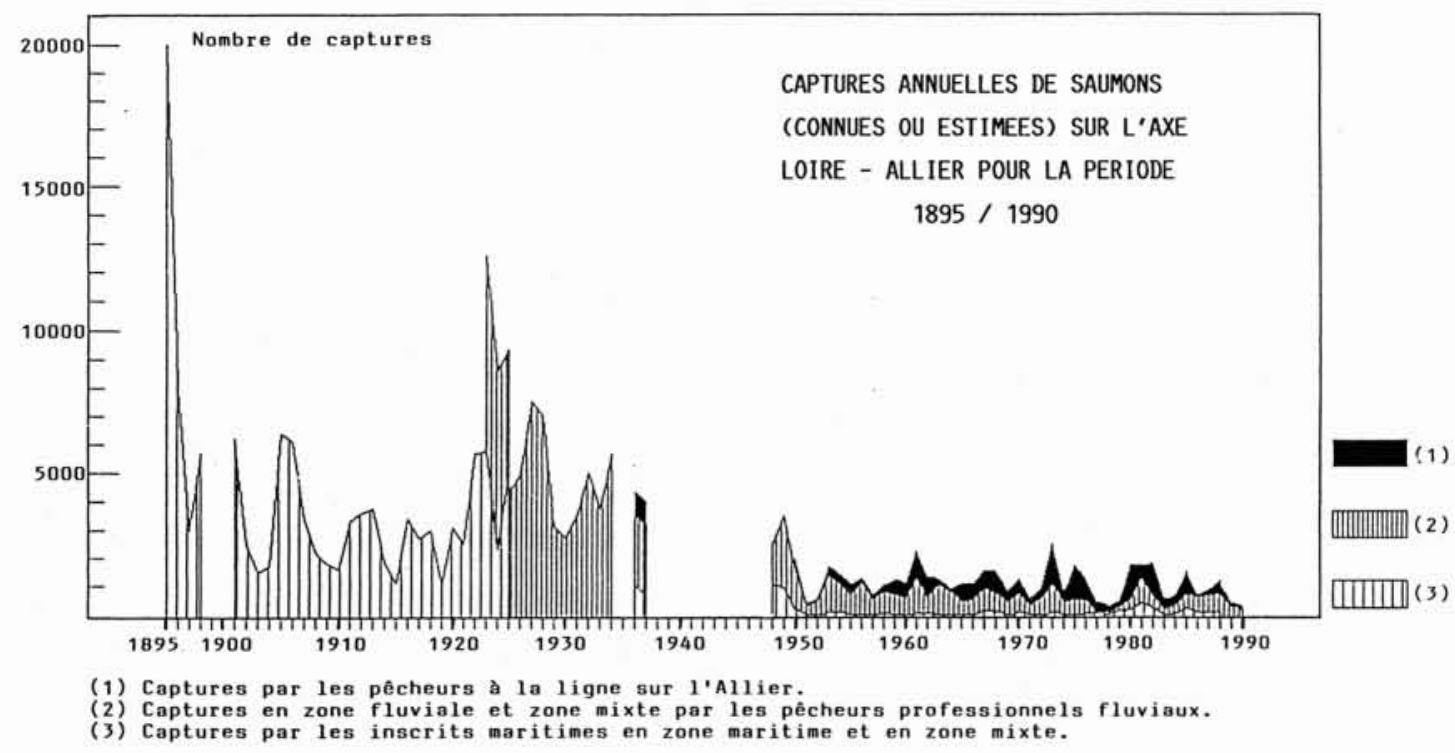

\section{Les actions entreprises depuis 1975, au siècle dernier. Les captures}

Au siècle dernier, les captures annuelles de saumons sur l'ensemble du bassin de la Loire étaient de l'ordre de la centaine de milliers. Elles ont progressivement diminué, pour varier entre un et trois milliers depuis 1950. En 1989, 90 et 91 , elles ont été nettement inférieures à un millier*.

La plus grande partie des captures (entre 60 et $80 \%$ selon les années) se fait aux filets, en estuaire et en Loire. Sur l'Allier, où on ne pêche le Saumon qu'à la ligne, les pêcheurs sont de plus en plus insatisfaits de cette situation. Il est certain qu'à une époque où la ressource est devenue très limitée, et où l'on sait élever, en cages marines, d'excellents saumons pour la consommation**, il serait plus rationnel de réserver une plus grande part des sujets sauvages à la pêche de loisirs, dont les retombées touristiques et économiques sont incomparablement plus importantes.

Beaucoup d'efforts ont pourtant été déployés en faveur des poissons migrateurs, et en particulier du Saumon et de son milieu, par le Ministère de l'Environnement, le C.S.P. et les collectivités de pêcheurs, dans les quinze dernières années.

La plus grande partie des ouvrages qui gênaient la migration a été équipée de passes à poissons. Seuls les barrages de Vichy et de Poutès-Monistrol (Haute-Loire) posent encore des problèmes sérieux, qu'il est d'ailleurs prévu de résoudre prochainement.

Des progrès ont également été faits pour les repeuplements : La Salmoniculture du C.S.P. à Augerolles (Puyde-Dôme) maîtrise maintenant la production d'œufs de souche pure Allier, ce qui lui permet de déverser annuellement plus de 50000 pré-saumoneaux, sans entamer sensiblement le cheptel de reproducteurs sauvages et sans avoir recours à des œufs d'origine étrangère, peu adaptés à nos cours d'eau. Ces sujets de repeuplement constituent, 25 à $50 \%$ (selon les années) du contingent total dévalant vers la mer.

La période d'ouverture de la pêche a été réduite, et des réserves instituées dans les zones sensibles, afin de restreindre les captures et préserver les reproducteurs.

Enfin, grâce aux aides de l'Agence de Bassin, et au soutien des débits estivaux par le Barrage de Naussac, la qualité de l'eau de l'Allier est restée en général correcte, du moins en amont de l'agglomération clermontoise; à l'amont de Brioude, elle est tout à fait propice à la reproduction du Saumon et à la vie des juvéniles.

Le regain de reproducteurs sur les frayères permettait d'espérer une nette augmentation du niveau de population à partir de 1980. En réalité, on doit constater maintenant que si la disparition de l'espèce a été évitée, l'augmentation ne s'est pas produite. Les contingents semblent même plutôt en diminution, en 1989, 90 et 91 .

\footnotetext{
* On n'a aucun moyen d'évaluer le contingent de saumon entrant chaque année en estuaire ou en Loire. On peut seulement avoir une idée de son évolution à partir de divers indices, notamment :

(a) comptage des nids de fraye, en novembre-décembre ;

(b) déclarations de captures; sur ce point, les statistiques « officielles " n'ont qu'une valeur très limitée car :

- les captures en estuaire et autour de l'estuaire sont très mal connues,

- les pêcheurs au filet, en Loire, qui ne déclaraient généralement qu'une partie de leurs prises ont maintenant tendance à en surdéclarer ;

- les pêcheurs aux lignes, qui déclaraient assez fidèlement leurs prises lors de la mise en place du sytème, le font moins bien depuis peu.

** Mis au point progressivement depuis 1970, ces élevages ont produit, en 1990, 224000 tonnes (environ 60 millions de poissons) de saumon atlantique, dont $70 \%$ proviennent de Norvège. Soit environ 50 fois plus que les captures de saumons sauvages de
} l'Atlantique la même année. 


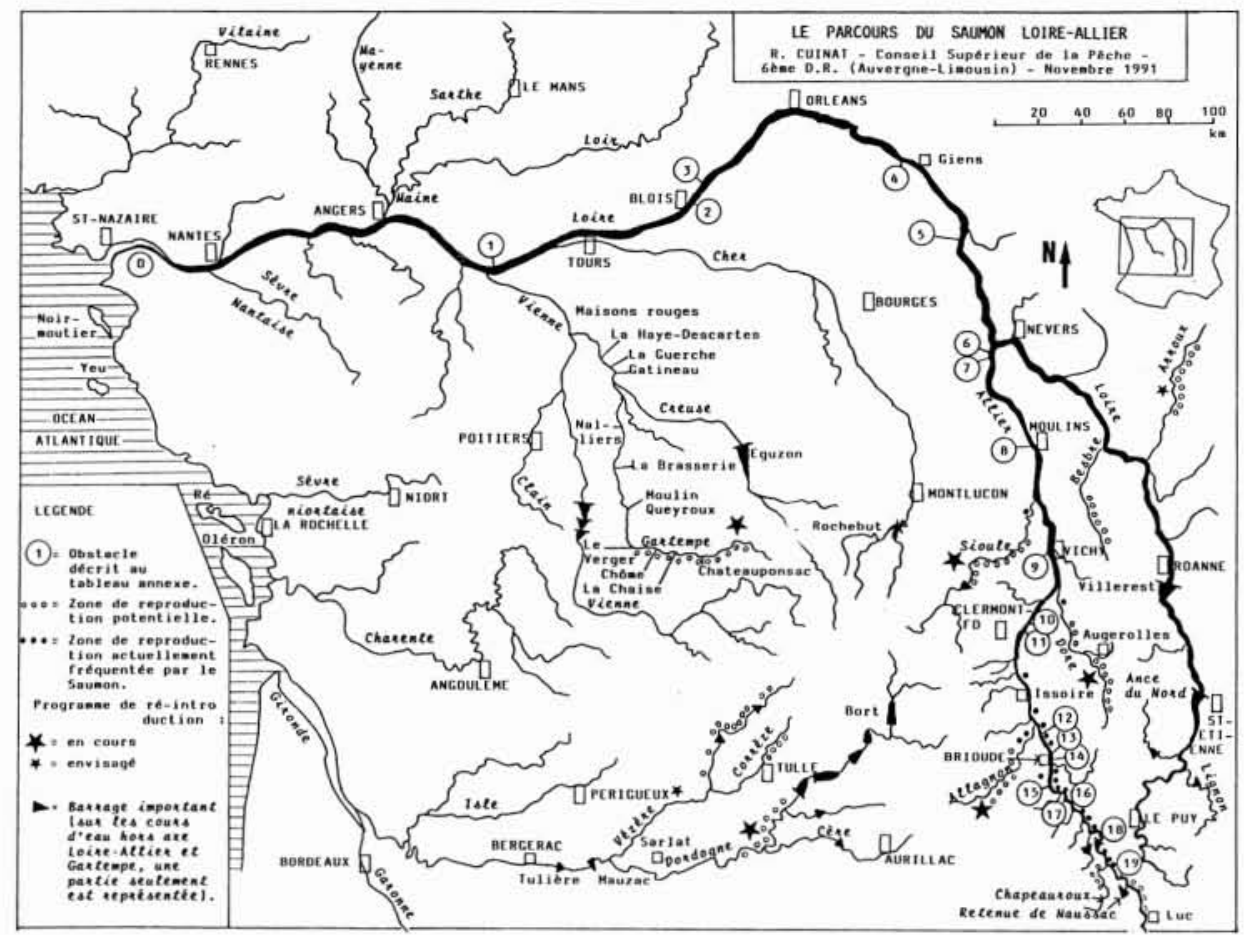

Le parcours du saumon Loire-Allier : situation en 1991

\begin{tabular}{|c|c|c|c|c|c|c|}
\hline \multirow[b]{2}{*}{$\begin{array}{l}\text { Situa- } \\
\text { tion } \\
\text { sur la } \\
\text { carte }\end{array}$} & \multirow[b]{2}{*}{$\begin{array}{l}\text { Désignation de } \\
\text { l'obstade }\end{array}$} & \multirow[b]{2}{*}{ Usage } & \multirow{2}{*}{$\begin{array}{l}\text { Hauteur } \\
\text { de chute } \\
\text { approxi- } \\
\text { mative } \\
\text { (m) }\end{array}$} & \multicolumn{2}{|c|}{ Passage des migrateurs } & \multirow[b]{2}{*}{$\begin{array}{l}\text { Améliorations } \\
\text { nécessaires }\end{array}$} \\
\hline & & & & $\begin{array}{c}\text { Dispositif } \\
\text { de } \\
\text { franchissement }\end{array}$ & $\begin{array}{c}\text { Problèmes } \\
\text { pour les } \\
\text { migrateurs } \\
(9\end{array}$ & \\
\hline 0 & $\begin{array}{l}\text { Bouchon vaseux aggravé } \\
\text { par les pollutions } \\
\text { et les dragages }\end{array}$ & & & & $3 \mathrm{md}$ & $\begin{array}{l}\text { oui, important } \\
\text { et urgent }\end{array}$ \\
\hline 1 & Seuil de Chinon & Centrale nucléaire & 0,5 & non & 1 & \\
\hline 2 & Barrage mobile de Blois & Retenue loisirs & 3 & $\begin{array}{c}\text { oul } \\
\text { (non fonctionnel) }\end{array}$ & $2 \mathrm{~m}$ & oui = à l'étude \\
\hline 3 & $\begin{array}{l}\text { Seuil de Saint-Laurent- } \\
\text { des-Eaux }\end{array}$ & Centrale nudéaire & 0,5 & non & $1 \mathrm{~m}, 2 \mathrm{~d}$ & oui $=$ prévu \\
\hline 4 & $\begin{array}{l}\text { Seuil de Dampierre-en- } \\
\text { Burly }\end{array}$ & Centrale nudéaire & 1 & non & $2 \mathrm{~m}$ & oui $=$ prévu \\
\hline 5 & Seuil de Belleville & Centrale nucléaire & 2 & $\begin{array}{l}2 \text { passes à fentes } \\
\text { verticales }\end{array}$ & 1 & \\
\hline 6 & Seull du Pont-du-Guetin & $\begin{array}{l}\text { Radier du Pont- } \\
\text { canal }\end{array}$ & 1,5 & $\begin{array}{l}2 \text { pré-barrages } \\
\text { partiels }\end{array}$ & 1 ou 2 & $\begin{array}{l}\text { à vérifier (équi- } \\
\text { pement récent) }\end{array}$ \\
\hline 7 & Barrage des Laurins & $\begin{array}{l}\text { Prise d'eau pour } \\
\text { canal }\end{array}$ & 1,2 & $\begin{array}{c}\text { oui } \\
\text { (peu fonctionnel) }\end{array}$ & 1 & \\
\hline 8 & $\begin{array}{l}\text { Seuil à Règemortes, } \\
\text { à Moulins }\end{array}$ & Radier de pont & 1,5 & $\begin{array}{l}\text { oui (2 pré-barrages } \\
\text { partiels) }\end{array}$ & 1 ou 2 & éventuellement \\
\hline 9 & Barrage mobile de Vichy & Retenue Ioisirs & 5 & $\begin{array}{l}\text { oul }=2 \text { passes a } \\
\text { ralentisseurs }\end{array}$ & 2 à 3 & oui $=$ important \\
\hline 10 & $\begin{array}{l}\text { Seuil marneux des } \\
\text { Madeleines }\end{array}$ & Néant & 2 & oul $=$ rustique & 1 & \\
\hline 11 & $\begin{array}{l}\text { Barrage mobile de } \\
\text { Pont-du-Chateau }\end{array}$ & Retenue loisirs & 0,8 & $\begin{array}{l}\text { oul }=2 \text { passes } \\
\text { à ralentisseurs }\end{array}$ & 1 & \\
\hline 12 & Barrage de Vézezaxx & $\begin{array}{l}\text { Ancienne prise } \\
\text { of eau }\end{array}$ & 1 & non & 1 à 2 & oui, envisagé \\
\hline 13 & $\begin{array}{l}\text { Barrage de La Bajasse, } \\
\text { à Brioude }\end{array}$ & $\begin{array}{l}\text { Prise d'eau et } \\
\text { loisirs }\end{array}$ & 2,5 & $\begin{array}{l}\text { oui passe et } \\
\text { piège de controle }\end{array}$ & 1 & \\
\hline 14 & $\begin{array}{l}\text { Barrage de Viellle- } \\
\text { Brioude }\end{array}$ & Microcentrale & 2 & partiel & 2 & oui \\
\hline 15 & Barrage de Chilhac & Moulin & 1 & non & 1 à 2 & oul \\
\hline 16 & $\begin{array}{l}\text { Barrage de Chambon- } \\
\text { de-Cerzat }\end{array}$ & Microcentrale & 2 & $\begin{array}{l}\text { oul }=\text { pré-barrage } \\
\text { partiel }\end{array}$ & 1 & \\
\hline 17 & Barrage de Langeac & Microcentrale & 2,5 & $\begin{array}{l}\text { oui }=2 \text { passes } \\
\text { à bassins }\end{array}$ & 1 ou 2 & oul \\
\hline 18 & $\begin{array}{l}\text { Barrage de Poutes } \\
\text { et usine de Monistrol }\end{array}$ & Hydro-électricité & 15 & $\begin{array}{l}\text { oui }=\text { ascenseur } \\
\text { et glissiere au } \\
\text { barrage }\end{array}$ & $3 \mathrm{~m}, 2 \mathrm{~d}$ & $\begin{array}{l}\text { oui, nécessaire } \\
\text { aussi d’atténuer } \\
\text { édusées de } \\
\text { l'usine }\end{array}$ \\
\hline 19 & $\begin{array}{l}\text { Barrage de } \\
\text { Saint-Etienne-du-Vigan }\end{array}$ & Hydro-électricité & 15 & $\begin{array}{l}\text { Barrage de Saint- } \\
\text { Etienne-du-Vignon }\end{array}$ & 4 & $\begin{array}{l}\text { oui = permet- } \\
\text { trait la réaver- } \\
\text { ture de } 15 \text { a } \\
20 \mathrm{~km} \text { de fraye- } \\
\text { res }\end{array}$ \\
\hline
\end{tabular}

(*) 1 = Franchissement presque toujours sans probleme. $2=$ Difficultes occasionnelles. $3=$ Difficultes frequentes, retards sensibles a la migration. $4=$ Franchissement impossible. Problemes principalement pour la montee $=\mathrm{m}$; la descente $=d$; les deux $=$ md. 


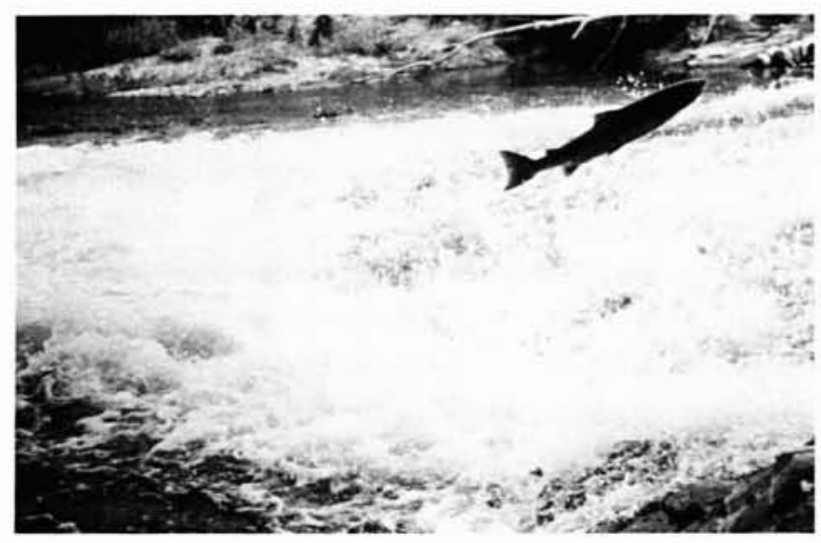

Un saumon tentant de franchir un barrage sur l'Allagnon. Cliché R. Cuinat (CSP-DR6)

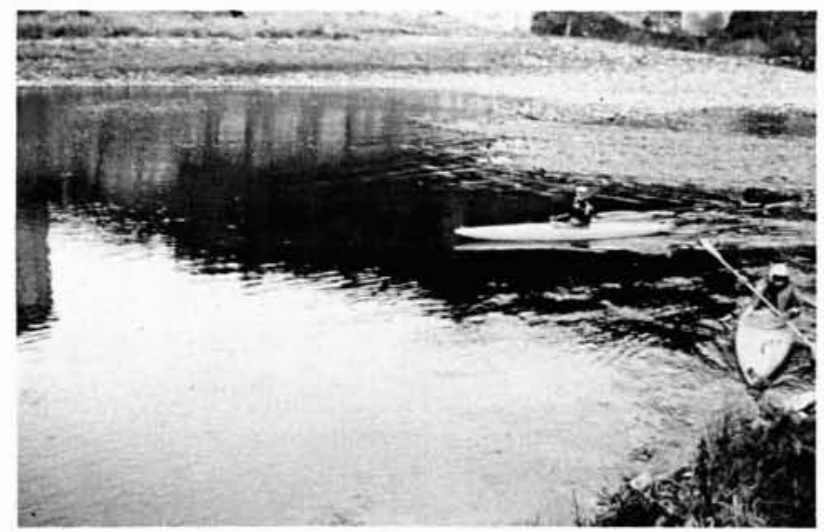

Repérage et comptage des "nids" de saumons sur le Haut-Allier. Cliché R. Cuinat (CSP-DR6).

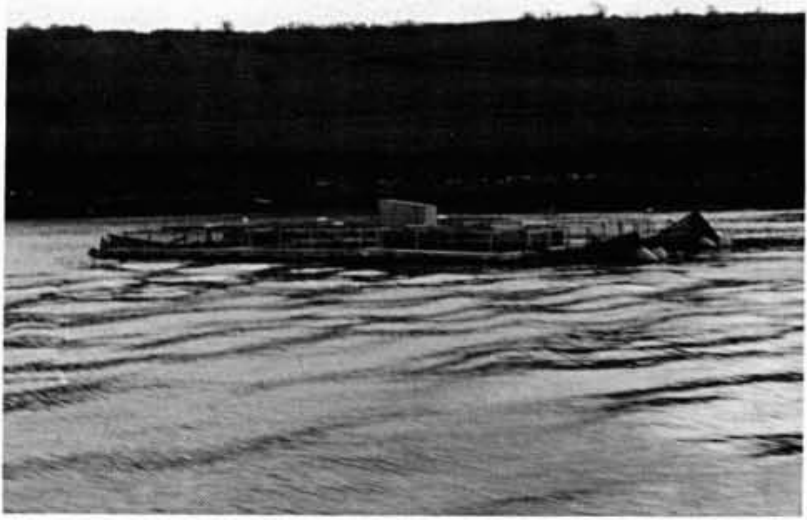

Elevage de saumons de consommation en " cages marines ", dans un fjord de Norvège. Cliché R. Cuinat (CSP-DR6).

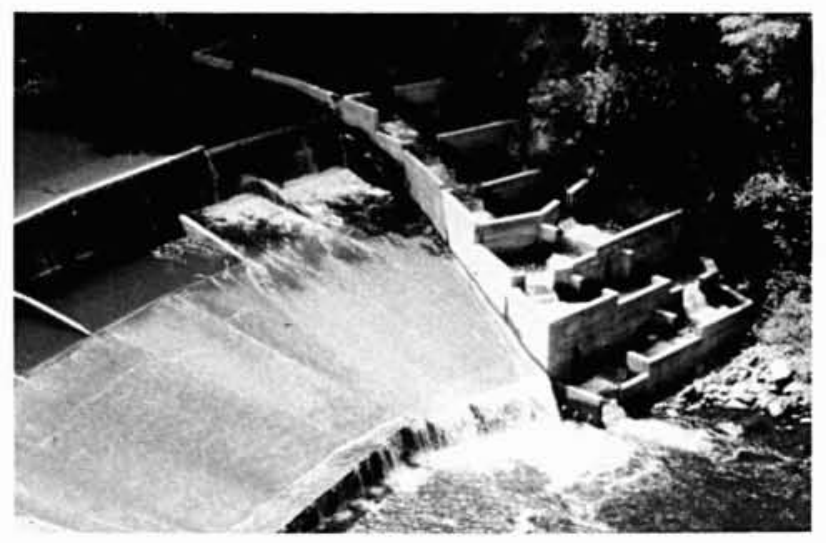

Echelle à poissons à bassins successifs sur un barrage de l'Allagnon. Cliché CSP-DR6.

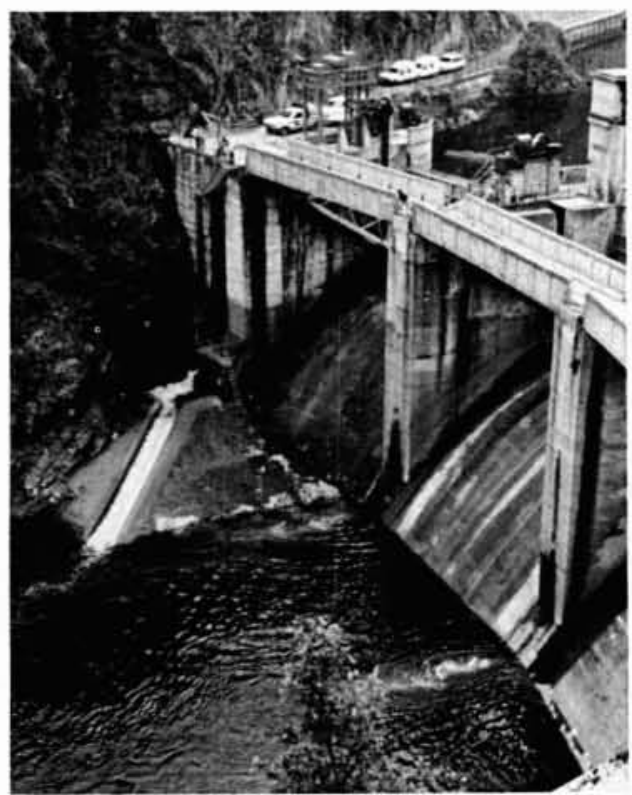

Ascenseur à poissons sur le barrage de Poutès-Monistrol, sur le Haut-Allier. Cliché P. Bomassi (CSP-DR6).

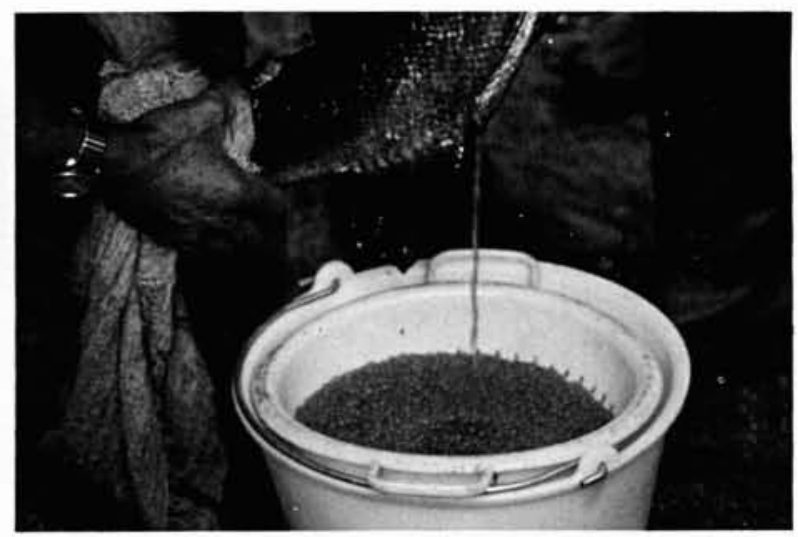

Ponte artificielle de Saumon pour le repeuplement, à la Salmoniculture d'Augerolles. Cliché R. Cuinat (CSP-DR6). 


\section{Comment peut-on expliquer cette situation?}

a) Pour l'ensemble de l'Atlantique, qu'il s'agisse des saumons nord-américains ou européens, les captures tendent globalement à diminuer depuis une vingtaine d'années ( $\cong 12000$ tonnes en 1972,4500 tonnes en 1990). Les conditions de croissance et de survie en mer, qui dépendent beaucoup des facteurs climatiques et sont très variables d'une année à l'autre, jouent certainement un rôle; il est probable, aussi que l'augmentation importante, dans la même période, des populations de phoques contribue à cette régression.

b) Les pêcheries en mer, notamment au large du Groënland et des Iles Féroës, continuent à prélever des saumons. Pour la plupart des pays atlantiques "producteurs", ces prélèvements apparaissent de plus en plus irrationnels, sachant qu'ils portent sur des sujets dont la croissance n'est pas achevée, et que l'élevage en cage suffit maintenant largement à satisfaire le marché.

Les négociations internationales, menées au sein du NASCO (National Atlantic Salmon Conservation Organization) ont conduit à des quotas (actuellement 900 tonnes au Groënland et 600 tonnes aux Féroës); elles permettent d'espérer un arrêt total des pêches aux Féroës; par contre, les Groënlandais, qui ont découvert cette ressource dans les années 60 , tiennent à la conserver, et la solution n'est pas encore en vue.

D'après nos marquages, près de la moitié des captures de saumons Loire-Allier a lieu au Groënland. Si ces prélèvements ne suffisent pas à détruire cette population, ils contribuent incontestablement à la fragiliser et à réduire les possibilités d'exploitation en eau douce.

c) Des problèmes graves sont apparus récemment en estuaire. Les dragages intensifs, joints à l'accroissement de la pollution, y ont créé une véritable barrière biologique. Ce problème s'est révélé brutalement à l'occasion des sècheresses exceptionnelles des années 89,90 et 91 , sècheresses aggravées par l'augmentation des prélèvements d'eau, en nappes et en rivières, sur l'ensemble du bassin. D'où provient cette transformation de l'estuaire, et pourquoi menace-t-elle non seulement le Saumon Loire-Allier mais tous les migrateurs du bassin de la Loire? C'est une question vaseuse, que nous tentons de résumer clairement au chapitre suivant.

\section{Que se passe-t-il en estuaire ?}

Les poissons migrateurs amphibiotiques ont besoin, pour passer de l'eau douce à l'eau salée et inversement, d'une phase d'acclimatation progressive, où leur organisme régule sa pression osmotique, et s'adapte à des conditions de vie et d'alimentation nouvelles. C'est en estuaire, vaste zone tampon où se brassent l'eau du fleuve et celle de la mer. que peut s'effectuer cette transition; chez les jeunes saumons, elle se déroule normalement sur quelques semaines, à une période très précise de l'année où leur organisme est prêt au changement (cette "fenêtre physiologique " ne dure que deux ou trois semaines).
Or, l'estuaire de la Loire est maintenant soumis à d'importantes modifications, qui se sont accélérées de façon inquiétante ces dernières années. Essayons de les résumer :

Pour permettre la remontée de gros navires, le port autonome de Nantes-Saint-Nazaire a créé, par dragage, un chenal de navigation d'une profondeur d'environ $13,5 \mathrm{~m}$ jusqu'à Donges-Montoir, et de $5 \mathrm{~m}$ jusqu'à Nantes. Pour réduire le coût, on emploie de plus en plus la méthode de dragage "à l'américaine " : la vase retirée du fond est aussitôt rejetée à l'eau afin que le courant la disperse. On pourrait la transporter au large, où elle jouerait le rôle d'un fertilisant, mais ce transport augmenterait les coûts. C'est pourtant ce qu'il faudrait absolument faire, compte tenu des très graves nuisances des pratiques actuelles :

1. L'estuaire est déjà excessivement pollué, d'une part du fait de l'eutrophisation de la Loire et des masses de plancton qui meurent et pourrissent en arrivant en eau saumâtre ; d'autre part à cause des égoûts de l'agglomération nantaise (plus de 800000 équivalents-habitants, qui ne sont encore épurés qu'à 25 pour cent environ). En $y$ ajoutant en permanence les produits du dragage, on augmente encore l'importance du "bouchon vaseux", sorte de soupe constituée maintenant par près d'un million de mètres-cubes de vase en suspension.

Dans ce bouchon vaseux, la teneur en oxygène est très faible ; elle est même tombé plusieurs fois à zéro au cours des trois dernières années; or, l'IFREMER et l'Université de Nantes, qui s'inquiètent aussi de cette dégradation, considèrent qu'aucune espèce de poisson ne peut survivre au-dessous de $3 \mathrm{mg} / \mathrm{l}$; des mortalités très importantes de muges ont d'ailleurs été observées ces dernières années.

2. L'approfondissement du chenal et le rejet direct des produits de dragage conduisent à des importants dépôts dans d'autres parties de l'estuaire, qui tendent maintenant à se combler. Les nouvelles terres ainsi conquises deviennent des prairies, gérées par le Port autonome et le «Syndicat des marais ".

Cette "poldérisation" accélérée inquiète beaucoup les pêcheurs, car l'estuaire constitue une nurserie irremplaçable pour beaucoup d'espèces de poissons, estuariens et marins.

3. Le comblement de l'estuaire, associé au surcreusement du chenal, bouleverse aussi la dynamique des échanges entre fleuve et mer: les eaux salées remontent depuis quelques années, avec les marées, en amont de la ville de Nantes, ce qui compromet son approvisionnement en eau douce. Le bouchon vaseux qui s'étend maintenant sur une très grande distance (plusieurs dizaines de kilomètres) fait de même, surtout lorsque le débit de la Loire est faible ; par débit moyen, il évolue entre Paimboeuf $(45 \mathrm{~km}$ aval de Nantes) et Saint-Nazaire. Il faut maintenant des débits supérieurs à $1000 \mathrm{~m}^{3} / \mathrm{s}$ pour qu'il soit repoussé vers l'estuaire externe, (Saint-Nazaire - Pornichet - Saint-Brévin).

4. Le canal de la Martinière, qui permettait de stocker de l'eau pour l'irrigation, est lui aussi en voie de comblement par la vase; et il devient de plus en plus difficile de l'alimenter en eau douce, du fait de la remontée du front de salinité. 


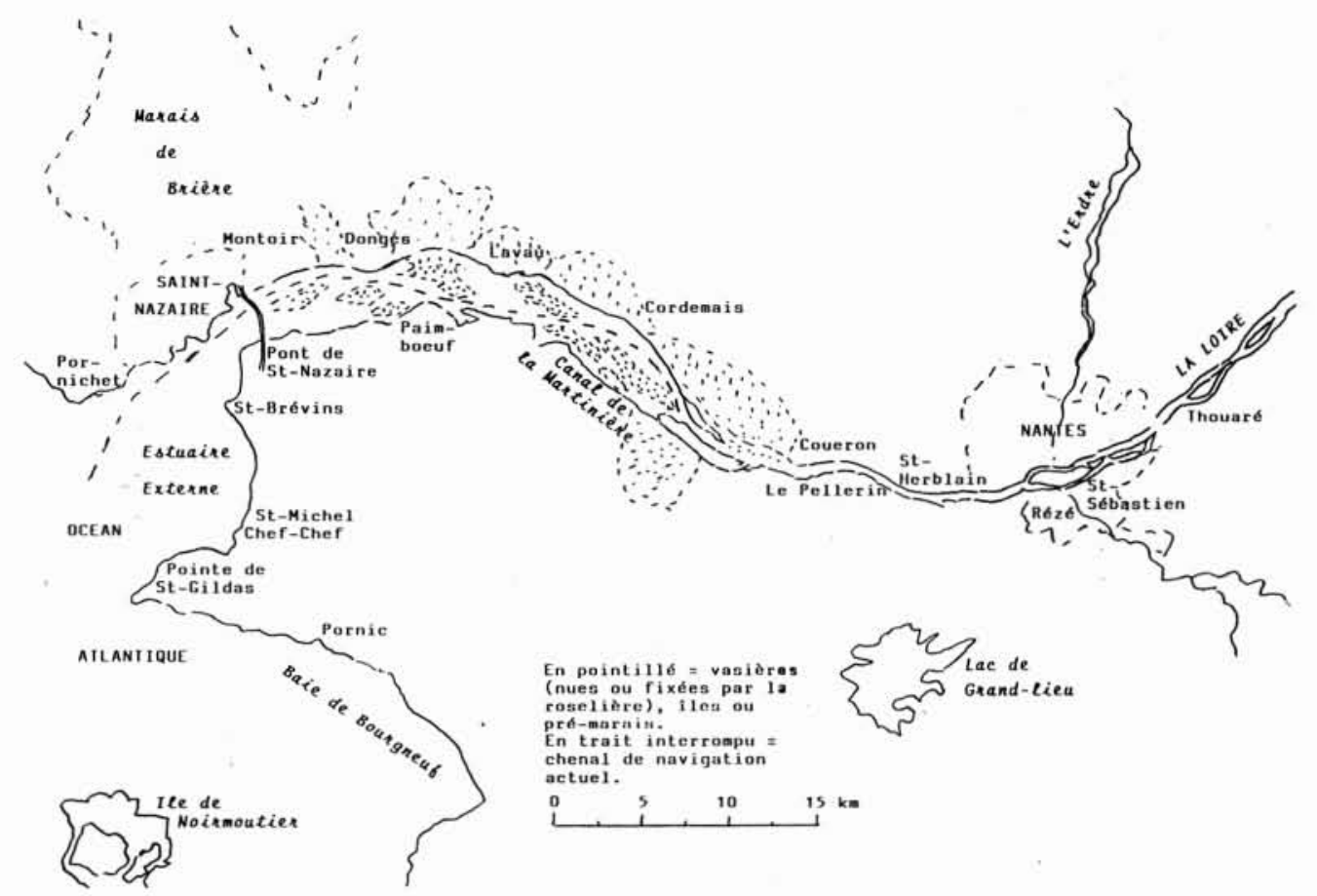

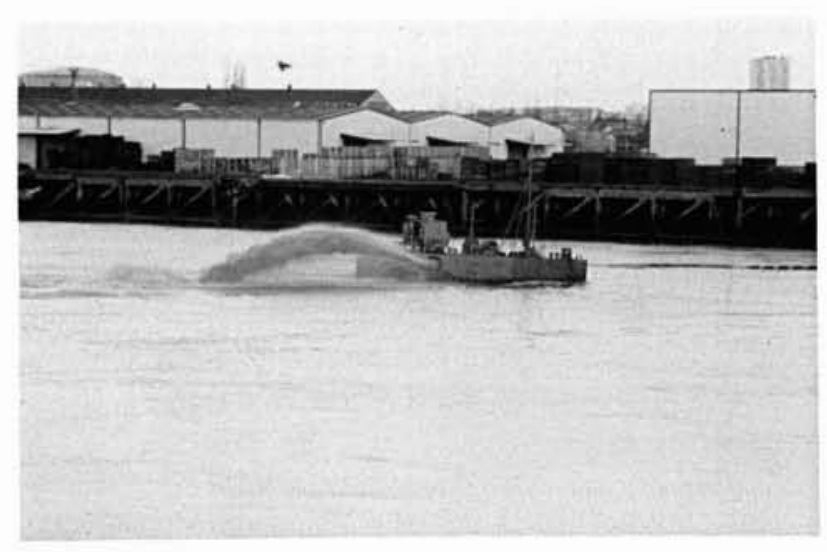

Dragage "à l'américaine » pour approfondissement du chenal de navigation dans l'estuaire de la Loire à Cheviré. Cliché J. Baudet.

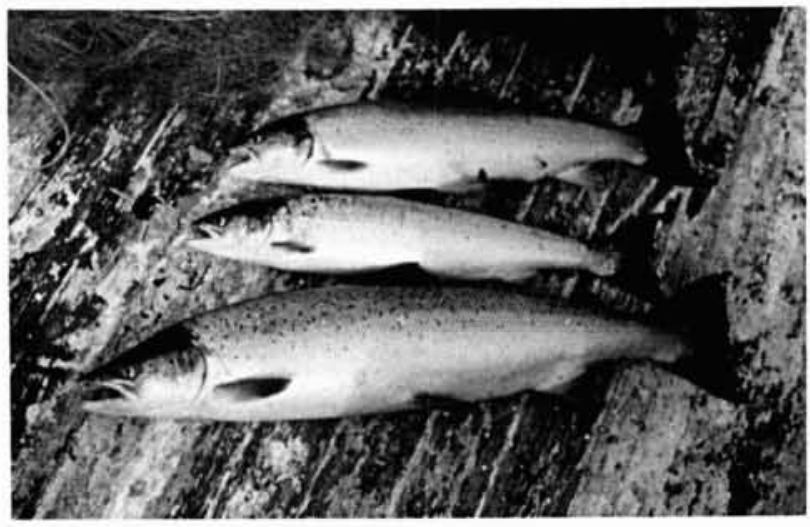

Saumons et truite de mer (au dernier plan) bloqués par le bouchon vaseux et capturés au filet en partie aval de l'estuaire. Cliché R. Cuinat (CSP-DR6).
Ainsi, c'est tout le système hydraulique du Sud-Loire qui est maintenant bouleversé, suite aux dragages inconsidérés du chenal.

5. Pour les poissons migrateurs, le bouchon vaseux constitue maintenant une barrière biologique infranchissable par débit normal.

Pour les saumons juvéniles, arrivant normalement en mai-juin dans cette zone, il est probable que le passage vers la mer est de plus en plus aléatoire.

Pour les adultes, les dommages sont certains : s'ils s'engagent en estuaire par débit normal, tentant de profiter d'une marée, ils seront soit refoulés, soit asphyxiés par le bouchon vaseux. Des dizaines de saumons morts ont ainsi été observés de 1989 à 91, ce qui n'avait jamais été vu auparavant. S'ils restent en mer, attendant une crue, ils sont exposés à la capture par les filets de marins pêcheurs ou de plaisancier. Ils prennent de plus un retard tel qu'ils ne pourront plus parvenir en temps voulu aux frayères du haut Allier ( $800 \mathrm{~km}$ en amont), et seront perdus pour la reproduction.

Ainsi, en 1990, les seules arrivées appréciables en Loire semblent s'être produites à l'occasion d'une crue en fin d'hiver, et se sont pratiquement arrêtées ensuite. En 1991, des saumons essayaient encore de remonter à Paimboeuf en fin juillet, alors qu'en année normale, les arrivées en Loire commencent en automne et sont terminées à la fin du printemps.

Il est certain que si les rejets polluants ne sont pas réduits et si les méthodes actuelles de dragage se poursuivent, la vie aquatique en estuaire deviendra de plus en plus aléatoire, et les populations de poissons migrateurs de l'ensemble du bassin de la Loire seront fortement compromises, le Saumon étant l'espèce la plus menacée de disparition. 


\section{LE SAUMON LOIRE-ALLIER}

\section{Les projets actuels d'aménagement de l'estuaire}

Le Port autonome, en vue de créer une nouvelle zone industrielle à Donges-Est, projette d'approfondir une partie du chenal; et d'y provoquer un auto-curage en concentrant le courant au moyen d'épis ou digues submersibles.

Les pêcheurs professionnels et les associations de protection de la nature sont très inquiets, les dépôts risquant d'être accentués dans toute la partie Sud de l'estuaire, en aval de Paimbœuf, ce qui accélérerait encore sa poldérisation.

De grosses études ont été entreprises sur ces projets et leurs conséquences, les mécanismes hydrauliques et sédimentologiques en jeu étant très complexes. Malheureusement, les effets sur le bouchon vaseux, sa concentration en "crême de vase", son étendue, ses déplacements et ses effets sur les poissons n'ont, à notre connaissance, pas été suffisamment pris en compte. Et surtout, la dimension "poissons migrateurs" des travaux actuels ou des projets d'aménagement dans l'estuaire, par le Port autonome de Nantes-Saint-Nazaire, a été manifestement sous-estimée jusqu'à présent. Il est plus que temps de s'en préoccuper très sérieusement, si l'on ne veut pas atteindre une situation irréversible, dont les conséquences intéressent une grande partie du bassin fluvial.

\section{Quel avenir pour les migrateurs du bassin de la Loire?}

Malgré tous les efforts déployés sur la Loire et ses affluents depuis quinze ans en faveur des espèces migratrices, certaines sont-elles menacées de disparition du fait de la dégradation de l'estuaire?

A court terme, je ne le pense pas. En effet:

- on peut espérer que les sécheresses que nous venons de connaître ne se renouvelleront pas aussi fréquemment que depuis 1989 ;

- et ces sécheresses elles-mêmes n'ont pas supprimé toute migration : des aloses et quelques saumons ont pu remonter la Loire chacune de ces trois années, et toute reproduction n'a pas été supprimée : ces poissons ont pu profiter de quelques "créneaux ", où de petites crues, conjuguées à des marées et des températures favorables, leur ont permis de franchir le bouchon vaseux.
Mais combien d'autres migrateurs sont-ils morts, soit d'asphyxie, soit de maladies liées aux températures et pollutions excessives, soit capturés par des filets à l'occasion des longues périodes d'attente forcée en estuaire ou en mer? Impossible de le savoir. Toutefois tous les indices montrent que les pertes, qu'elles soient dues aux mortalités ou aux retards de migration, sont très importantes, en tout cas pour le Saumon.

A moyen terme, par contre, cette disparition est fort à craindre, selon le scénario pernicieux suivant:

Avec le barrage biologique en estuaire, tous les efforts déployés sur le bassin ne donneront que des résultats minables.

Le grand retour du Saumon que nous avions espéré 15 à 25000 poissons par an, grâce aux précédents "programmes migrateurs " et aux excellentes frayères du HautAllier et de certains affluents - restera un mythe inabordable; et surtout les programmes, coûteux et contraignants, de restauration du Saumon dans la Dore et la Sioule, dans la Loire supérieure et l'Arroux, et sur l'axe Vienne-Creuse-Gartempe seront bientôt assimilés à des publicités mensongères: on y verra bien revenir quelques poissons, mais si rares et si précieux que personne n'aura le droit d'y toucher. Et sans possibilité de pêche, ni retombées touristiques ou socio-économiques pour les régions qui avaient joué le jeu, qui défendra encore le Saumon et son milieu? Les naturalistes et les conservateurs de gènes feront-ils seuls le poids, face à la pression permanente de notre "civilisation" ? C'est peu probable. C'est alors que le Saumon Loire-Allier, et peut-être d'autres espèces avec lui, risque fort d'entrer dans l'Histoire.

Ainsi, la plus grave menace pour les poissons migrateurs - et en particulier le Saumon - du Bassin de la Loire réside maintenant dans le surcreusement de l'estuaire et l'expansion du bouchon vaseux qui en résulte.

On pouvait, jusqu'ici, "draguer par ignorance». Il est maintenant établi que l'estuaire de la Loire est le plus malade de tous les estuaires français. Non seulement les pêcheries en mer, mais les poissons migrateurs de l'ensemble du fleuve en subissent de lourdes conséquences.

Le diagnostic étant fait, va-t-on laisser l'intérêt local dominer l'intérêt général ? Permettre aux uns d'écraser les pieds pendant que les autres essaient de soigner le corps?

Espérons que la France saura faire le choix, et sauver à temps un patrimoine naturel sur un grand fleuve que l'Europe nous envie. 


\section{R. CUINAT}

\section{Bibliographie}

Anonyme, 1992. - Bouguenais-informations. Numéro spécial Estuaire de la Loire, février 1992 - Mairie de Bouguenais, 44340 Bouguenais.

SaUriau P.G., 1991. - Etude des populations de mulets dans l'estuaire de la Loire. Bilan des études 1989-1990 - Université de Nantes : 201 p.

ROMAÑA L.A. et al., 1990, - Influence du bouchon vaseux sur les variations des concentrations en oxygène dissous. Cas de l'estuaire de la Loire - La Houille Blanche, 3-4 : 257-263.

MARChAnd J., 1988. - Rôles de l'estuaire de la Loire vis-à-vis des espèces d'intérêt halieutique - Norois, 34 (133-135) : 379-390.

Sauriau P.G., Robin et Marchand J., 1991. - Les mortalités estivales du mulet Liza Ramada en Loire: dysfonctionnement cyclique du rôle de voie de migration d'un estuaire - Colloque U.O.F. Société d'Ecologie Nantes, juillet 1991 : $20 \mathrm{p}$.

Sıсот S., 1992. - La Loire qui pue. Le Pêcheur Professionnel, $\mathrm{n}^{\circ} 2,1^{\text {er }}$ trimestre $1992: 16-19$. 